\title{
Examining Patterning Abilities in First Grade Children: A Comparison of Dimension, Orientation, Number of Items Skipped and Position of the Missing Item
}

\author{
K. Marinka Gadzichowski \\ Department of Psychology, George Mason University, Fairfax, USA \\ Email: kgadicho@gmu.edu
}

Received July $18^{\text {th }}$, 2012; revised August 20 ${ }^{\text {th }}$, 2012; accepted September $16^{\text {th }}, 2012$

\begin{abstract}
Current curricula in most school districts in the United States include some instruction on the recognition of patterns in kindergarten and continuing into the early elementary school years. Despite the fact that patterning is so common in school cirricua, very few reports of what types of patterns are easy or difficult for children to learn have been published. In an effort to address this issue, 121 first grade children from an urban school district were tested with 48 patterns that varied in dimension, orientation, position of missing items, and magnitude of the gap between items. An ANOVA for completely correlated factors was conducted. Results indicated that only the magnitude of gaps (i.e., "skips") made a significant difference. There were indications of an interaction between that factor (skips) and the position of a missing item. Implications were discussed.
\end{abstract}

Keywords: Patterning; Oddity; Transitivity

\section{Introduction}

Instruction in recognizing patterns constructed from small objects, shapes, colors, and sometimes letters and numbers, begins in kindergarten and continues into the early elementary grades for most children enrolled in school in the United States. Several instruction manuals exist concerning how to conduct this instruction, which is called "patterning" (Burton, 1982; Ducolon, 2000; Jarboe \& Sadler, 2003). Patterning instruction occurs nationwide but is not founded on empirical research; rather, it is based on a consensus of educators who are of the belief that the ability to recognize such patterns holds some educational value (National Councils of Teachers of Mathematics, 1993). It is also commonplace in parts of Europe (Threlfall, 1999; Lilijedahl, 2004). The present way of thinking is that instruction in patterning improves abstract cognitive abilities (Economopolous, 1998; Papic, 2007) that ultimately will lead to improved academic performance.

Empirical investigation of this proposition is quite limited. There are only three experimental studies that address how this instruction impacts other cognition or achievement. In Herman's (1973) dissertation, 24 lessons on patterns made from shape, size and color were given to 71 kindergarten children from an impoverished background. The patterns were alternations of two or three items-AABAAB, ABBABB, ABABAB, $A A B B A A B B$, and ABCABC. Numeracy ability was subsequently measured by two subtests of the Metropolitan Readiness Test. Her findings were that English-speaking children who received this instruction made significant gains in numeracy skills following the instruction, although Spanishspeakers did not.

A multiple baseline study by Hendricks et al. (1999) used only four participants, all of whom were males in first grade who had begun to lag behind their peers. All four of the par- ticipants were English Language Learners. They were given instruction in both patterning and class-inclusion. Two of the four boys showed significant gains on the Slosson Intelligence Test (SIT), one made gains approaching statistical significance and one did not show a gain. The children all had low scores on each of the Diagnostic Achievement Battery-2 (DAB-2) subscales, which measured total achievement, listening, reading, speaking, writing, math, written and spoken language. Although individual gains were different there was an overall pattern showing that the gains on patterning and class inclusion were accompanied by significant gains on the DAB-2 measures of academic achievement. The shortcoming of this multiple baseline study is that while it shows that the children improved on what they were taught (patterning and class inclusion), it does not prove that gains on the SIT and DAB-2 was because of the instruction they had received. There are a number of reasons why an ESL child could make such gains. ESL children may come into the school system with very low capacity for English. Some ESL children are unable to communicate even a simple thought when they begin the school year. After working with their ESL teacher and spending seven hours a day hearing and attempting to speak English, it is quite easy to see that they would improve rapidly. Intelligence tests and measures of academic achievement rely on the ability to communicate in English. Children who have a poor vocabulary in English or do not yet understand fully what is being said are going to score lower as a result of their lack of language. As they learn new vocabulary during the school year and become comfortable expressing themselves in English it is only natural that they would score higher on such tests. Additionally, a child who does not comprehend what is being asked of him may choose to randomly select answers without even thinking about the choice, simply as an avoidance tactic. Once they have attained mastery of the 
English language they no longer feel insecure and are likely to contemplate the answer choices without being self-conscious.

In 2006, Hendricks, Trueblood, and Pasnak expanded the depth of patterning instruction to include 480 color, size, number, letter, and time patterns ranging from alternations similar to those of Herman (1973) to complex patterns with varying numbers of steps between items presented in matrices. The results from their study indicated that the children who received this type of instruction made greater academic gains than children in their control groups, who had received instruction focused specifically on academic material that had been developed upon the recommendation of teachers. The children's scores on the DAB Total Achievement measure were correlated with the patterning test scores, $r(60)=.40 p<.01$. Correlations for the DAB's subscales were similar: mathematics $r(60)=.42$, $p<.01$, written language, $r(60)=.35, p<.01$, spoken language, $r(60)=.38, p<.01$ These are considered to be medium effect sizes (Cohen, 1992).

There were no group differences on the DAB scale. However, in spite of random assignment of participants to groups, there was a 5 point difference between the groups on average intelligence, favoring the control group, and unequal variances. Because of this, a MANCOVA was conducted and the SIT-R scores were used as a covariate. Once the groups were equated statistically on the SIT-R, the patterning group did display improvement that was significantly more than the improvement made by the control group on the DAB Total Achievement measure.

These three studies constitute the entire set of empirical evidence that instruction in patterning has an effect beyond helping children become better at patterning per se. However, it is clear that patterning instruction has become a fixture in education, and for that reason deserves investigation.

To date, no research has been published in the professional literature that was designed to determine which types of patterns are the easiest or hardest for children to learn. However, Boyer, Sweeting, Pasnak and Kidd (2010) presented a poster indicating that the number of items a pattern "skipped" was relevant. First grade children were most accurate on patterns that skipped a single item, followed by patterns that skipped two items and finally, patterns that skipped three items.

For younger (preschool) children, research involving oddity problems showed that the dimension (color, form, size, and orientation) in which it was presented was relevant in determining ease or difficulty of an oddity problem. Color oddity problems were easier than form oddity and orientation oddity problems (Gadzichowski \& Pasnak, 2010). Since the dimension of the oddity problem did impact how easy the problem was for the younger children (age 4), it is an area of interest when assessing patterning ability in older children. Perhaps the dimension in which a pattern is presented affects how difficult it is to understand the pattern. Boyer, Sweeting, Kidd, and Pasnak (2010) reported that patterns of letters were easier for children than patterns of time as represented by clock faces, and patterns of numbers were harder than patterns of rotation, time and letters. However, their design was nonfactorial and incomplete, and Gadzichowski, Kidd, Pasnak, and Boyer (2010) reported that these dimensions did not produce significant differences, so the issue is only partially resolved.

Developmental psychologists have neglected patterning in their study of cognition. There is no theory that puts patterning among the cognitive tasks mastered during a child's develop- ment. Furthermore, there is no understanding or explanation of how failure to master this cognitive ability would detract from a child's ability to master more abstract cognitive concepts. However, children transition from one type of thinking in preschool to a more complex cognition in early elementary school-the transition from preoperational to concrete operational thought (Piaget, 1963/1936) — and patterning incorporates many of the changes in reasoning ability made during this time. Patterning may be closely related to transitivity, an ability in which someone is capable of deducing the relationship between two items by examining how those items relate to a third item. Clements and Sarama (2007) found that having the ability to make indirect comparisons, along with the ability to order objects according to their size (seriation) are cognitive skills that are necessary for successful performance in elementary school. "Indeed, research indicates that mastering these skills is one of the strongest predictors of school success or failure" (Clements \& Sarama, 2008: p. 365). In seriation, a child must understand how an item relates to the items that come before or after it in the sequence. When looking at patterns, this same rule applies. In seriation, the relation is a simple one. The item in question is either smaller or larger than the neighboring items on some dimension (height, width, weight, length, or overall size). In patterning the relation is more complex; the relation might involve size, color, size and color, shape, or other dimensions that are more abstract, and the relationships can be much more complicated.

Transitivity is the understanding that if item $\mathrm{A}$ is related to item B in some way, and item B is related to item C in some way, then the relationship between $\mathrm{A}$ and $\mathrm{C}$ can be determined by comparing these items to item $\mathrm{B}$. The relationship between A and C (the key items) is not directly observed but rather deduced by comparing the key items to another item. Both transitivity and patterning incorporate the idea that an item is defined by, and simultaneously defines, properties of items that follow or precede it. The primary difference between transitivity and patterning is that patterning does not require an individual to utilize the relations of $\mathrm{A}$ to $\mathrm{B}$ and $\mathrm{C}$ to $\mathrm{B}$ in order to determine the relation of A to $\mathrm{C}$. Transitivity does require that one use those relations. Since a child could make use of the transitive relation or alternatively make use of the simultaneous presentation of all the items and compare A to $\mathrm{C}$ directly, perhaps patterning is a precursor to transitivity.

Examining the differences between patterns wherein the missing item occurs at the beginning, middle or end may offer some insight into the relationship between patterning and transitivity. Children who are capable of solving patterns that are missing the beginning or end item may have begun to master transitivity, because they must infer the nature of the missing item from one that just precedes or follows it. When children can rely on the presence of items on both sides of a missing item (patterns missing a middle object) to determine the nature of the missing item, they may be reversing the transitivity operation. Thus, when a child understands patterns of items in which some items follow and precede other items, based on the rule of that pattern, then the child can make inferences about a neighboring item by looking at any one item in the pattern. A more advanced extrapolation would be to use a single item to make inferences about both of the neighboring items successively or simultaneously. The ability to compare the two inferences about the neighboring items in order to relate the neighboring items to one another would be considered transitivity. 
Hence, making inferences about neighboring items from pattern rules may be a step in the process of developing transitivity.

A first step in understanding patterning as an aspect of cognitive development is to determine what kinds of patterns are easy for children and what kinds are difficult. For this reason the research questions are as follows: Does the dimensioncolors, shapes, letters, etc.-make a difference, or do children abstract the pattern rule independently of the dimension in which it is presented? A second question involves the orientation of the pattern. Is a pattern presented horizontally, easier than a similar pattern presented vertically? Intuitively, it seems that there would be a preference for a particular orientation. Children read from left to right, but number problems are most often presented in a vertical orientation. Also of interest is the number of items "skipped" in a pattern. It seems likely that children find patterns that skip a single item to be easier than those patterns that skip multiple items. Finally, when a pattern is incomplete, does the position of the missing item make a difference? Answers to these questions, which have never been asked by psychologists or educators, can inform investigations of how patterning relates to children's cognitive development, and also aid educators in determining what types of patterns can form the basis for the most fruitful classroom instruction.

\section{Method}

\section{Participants}

After approval from an internal review board, parental consent was obtained for 121 first-grade children from an urban school district. Children took permission forms outlining the research home to their parents and parents had the option to return the permission form with their signature, allowing their child to participate in the study. There were 67 females and 54 male participants. Of those participants 52 (43\%) were African American, 43 (36\%) were Hispanic/Latino, 16 (13\%) were Middle Eastern, 3 (2\%) were Caucasian and 7 (6\%) were of an ethnicity other than those listed. In the school district from which participants were drawn, $57 \%$ of children receive free or reduced cost lunch.

\section{Materials}

The patterns used consisted of four items that were shown in a vertical or horizontal array, and one space where a missing item should be placed to complete the pattern consisting. The child was to select from four options the item which would properly complete the pattern.

There were 12 rotation patterns, 12 time patterns, 12 letter patterns and 12 number patterns. The rotation patterns consisted of an object such as a bird that was rotated clockwise into one of eight possible positions. One type of rotation problem featured a pattern that involved skipping single positions, e.g. the bird was rotated from right side up to being horizontal, having skipped being slanted at a 45 degree angle to the right. The second type of rotation problem featured the object in question skipping two positions, e.g. the bird started out right side up, then skipped the 45 degree angle as well as being horizontal and was presented at a 45 degree angle with its head facing the lower right hand side of the page.

The time problems were constructed using clock faces. One type of time problem showed clocks on which the time changed by one hour and the other type of problem showed clocks on which the time changed by two hours.

Letters were used to construct a third type of problem. The letters used were all capital letters and as with the previous problems there were letter problems that skipped a single letter, e.g., P, R, T etc., and problems in which two letters were skipped, e.g., B, E, H, etc.

Finally, the fourth type of problem was comprised of numbers 1 through 30 . The number patterns included problems that skipped a single number, e.g. 7, 9, 11 etc., as well as patterns that skipped two numbers, e.g., 12, 15, 18, etc.

All of the aforementioned patterns were presented with the missing item at the beginning, middle or end of the pattern. All patterns were presented horizontally as well as vertically (see Figure 1).

\section{Procedure}

The patterns were presented to participants using a three ring binder which held patterns that had been printed out in color, on paper, and then placed into plastic protectors. Participants sat on one side of the table and the researcher sat opposite them. The patterns were presented to each child individually, and each child had as much time as he or she needed to time to select the correct answer from four the possible options given. Because there was some concern about the attention span of the children participating, the 48 pattern problems were given over the course of two days; 24 problems were given the first day and 24 problems were given on the second day. The order of the presentation was counterbalanced by dimension, orientation and the number of items the pattern skipped. The position of the missing item was randomized within these constraints.

\section{Results}

Descriptive statistics are presented in Table $\mathbf{1}$ and the analysis is presented in Table 2. An ANOVA for completely correlated factors was conducted, as every subject provided data for every cell. There was a significant difference between patterns that skipped one item and patterns that skipped two items. There were no significant differences between the positions of the missing item in the patterns, and no significant differences between the four dimensions, or the two orientations. The interactions of these characteristics were also nonsignificant. There was a significant difference between patterns that skipped one item and patterns that skipped two items. However, the interaction of the number of items skipped and the position of the missing item approached statistical significance, making acceptance of the null hypothesis for this comparison especially risky.

\section{Discussion}

The finding that the four dimensions of time, rotation, letters and numbers did not differ significantly was not surprising and in fact supports a previous finding (Gadzichowski et al., 2010). In that research, overall structure of the pattern proved to more important than the type of items used in presentation. Although one can never accept the null hypothesis in complete confidence, in the case of patterning, it seems that the dimension in which it is presented does not really play a primary role in how easy or difficult a pattern is.

The fact that the orientation of the pattern (vertical presentation or horizontal presentation) was not significant is of 


\section{K. M. GADZICHOWSKI}

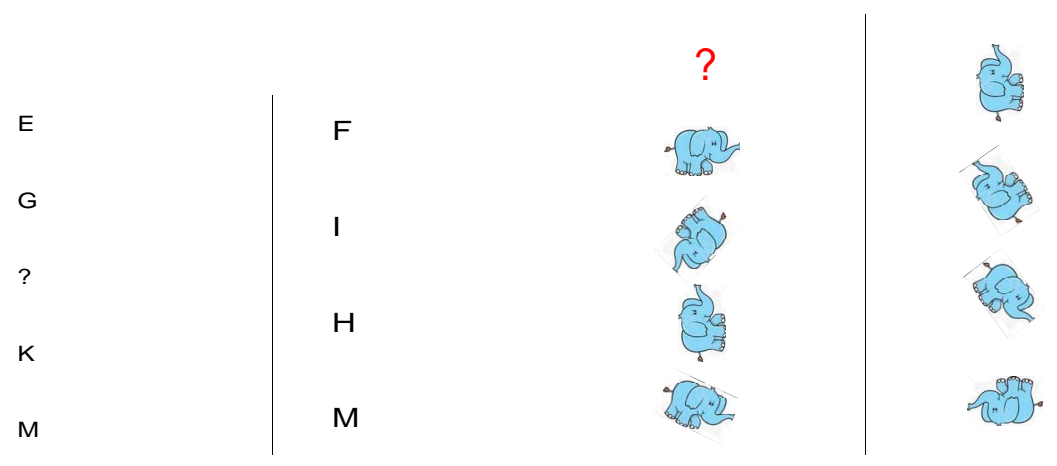

(a)

(b)
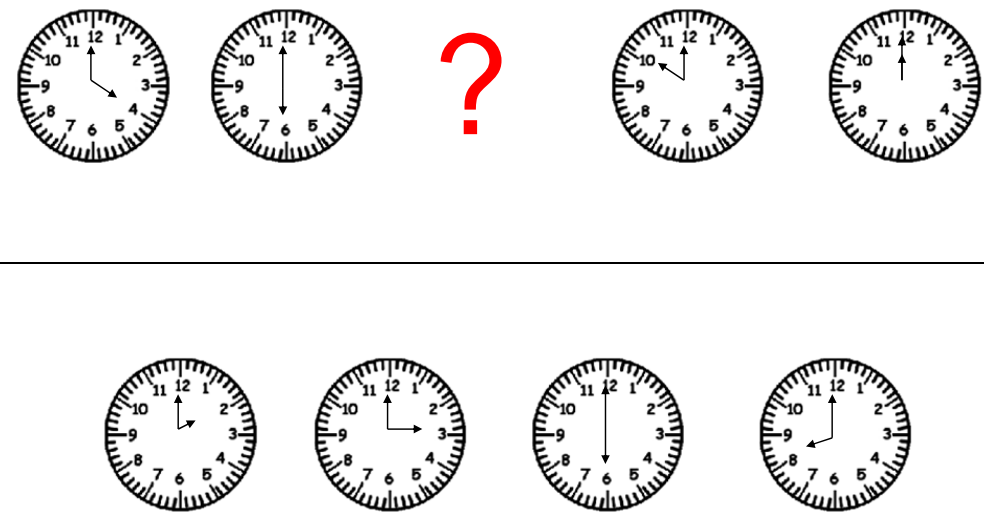

(c)

$\begin{array}{lllll}5 & 7 & 9 & 11\end{array}$

$12 \quad 13 \quad 15 \quad 10$

(d)

Figure 1.

The examples are (a) a letters pattern, presented vertically, skipping a single letter, the middle item is missing; (b) a rotation problem, presented vertically, skipping two positions, the missing item at beginning; (c) a time problem, presented horizontally, skipping one "item" (one hour of time), the middle item is missing; and (d) a numbers pattern presented horizontally, skipping a single number, the end item is missing. The layout for this figure required some distortions; the patterns shown the children were all equal in size.

Table 1.

Descriptive statistics for dimension, orientation, skip, and position.

\begin{tabular}{ccccc}
\hline Dimension & Rotation & Time & Letters & Numbers \\
\hline Mean & 1.528 & 1.534 & 1.529 & 1.529 \\
SE & .013 & .013 & .013 & .013 \\
Position & First & Middle & End & \\
Mean & 1.528 & 1.545 & 1.516 & \\
SE & .011 & .011 & .011 & \\
Orientation & Horizontal & Vertical & & \\
Mean & 1.528 & 1.531 & & \\
SE & .009 & .009 & & \\
Skip & One & Two & & \\
Mean & 1.544 & 1.516 & & \\
SE & .009 & .009 & & \\
\hline
\end{tabular}


Table 2.

ANOVA for dimension, orientation, skip, and position.

\begin{tabular}{|c|c|c|c|c|c|}
\hline Factor & $d f$ & MS & $F$ & $p$ & Partial Eta Squared \\
\hline Individuals (I) & & 120 & 360.09 & & \\
\hline Dimension (D) & 3 & .01 & .04 & $>.05$ & .00 \\
\hline $\mathrm{D} \times \mathrm{I}$ & 360 & .24 & & & \\
\hline Orientation (O) & 1 & .01 & .06 & $>.05$ & .00 \\
\hline $\mathrm{O} \times \mathrm{I}$ & 120 & .18 & & & \\
\hline Skip (S) & 1 & 1.38 & 4.76 & $<.05$ & .03 \\
\hline $\mathrm{S} \times \mathrm{I}$ & 120 & .29 & & & \\
\hline Position (P) & 2 & .34 & 1.70 & $>.05$ & .02 \\
\hline $\mathrm{P} \times \mathrm{I}$ & 120 & .20 & & & \\
\hline $\mathrm{D} \times \mathrm{O}$ & 3 & .15 & .88 & $>.05$ & .01 \\
\hline $\mathrm{D} \times \mathrm{O} \times \mathrm{I}$ & 360 & .17 & & & \\
\hline $\mathrm{D} \times \mathrm{S}$ & 3 & .16 & .80 & $>.05$ & .01 \\
\hline $\mathrm{D} \times \mathrm{S} \times \mathrm{I}$ & 360 & .20 & & & \\
\hline $\mathrm{D} \times \mathrm{P}$ & 6 & .11 & .55 & $>.05$ & .01 \\
\hline $\mathrm{D} \times \mathrm{P} \times \mathrm{I}$ & 720 & .20 & & & \\
\hline $\mathrm{O} \times \mathrm{S}$ & 2 & .11 & .92 & $>.05$ & .01 \\
\hline $\mathrm{O} \times \mathrm{S} \times \mathrm{I}$ & 240 & .12 & & & \\
\hline $\mathrm{O} \times \mathrm{P}$ & 1 & .15 & .88 & $>.05$ & .01 \\
\hline $\mathrm{O} \times \mathrm{P} \times \mathrm{I}$ & 360 & .17 & & & \\
\hline $\mathrm{S} \times \mathrm{P}$ & 2 & .44 & 2.59 & $<.10$ & .02 \\
\hline $\mathrm{S} \times \mathrm{P} \times \mathrm{I}$ & 240 & .17 & & & \\
\hline $\mathrm{D} \times \mathrm{O} \times \mathrm{S}$ & 3 & .13 & .72 & $>.05$ & .01 \\
\hline $\mathrm{D} \times \mathrm{O} \times \mathrm{S} \times \mathrm{I}$ & 360 & .18 & & & \\
\hline $\mathrm{D} \times \mathrm{O} \times \mathrm{P}$ & 6 & .45 & 2.04 & $>.05$ & .02 \\
\hline $\mathrm{D} \times \mathrm{O} \times \mathrm{P} \times \mathrm{I}$ & 720 & .22 & & & \\
\hline $\mathrm{D} \times \mathrm{S} \times \mathrm{P}$ & 6 & .29 & 1.61 & $>.05$ & .01 \\
\hline $\mathrm{D} \times \mathrm{S} \times \mathrm{P} \times \mathrm{I}$ & 720 & .18 & & & \\
\hline $\mathrm{O} \times \mathrm{S} \times \mathrm{P}$ & 2 & .35 & .37 & $>.05$ & .01 \\
\hline $\mathrm{O} \times \mathrm{S} \times \mathrm{P} \times \mathrm{I}$ & 240 & .19 & & & \\
\hline $\mathrm{D} \times \mathrm{O} \times \mathrm{S} \times \mathrm{P}$ & 6 & .51 & 1.11 & $>.05$ & .02 \\
\hline $\mathrm{D} \times \mathrm{O} \times \mathrm{S} \times \mathrm{P} \times \mathrm{I}$ & 720 & .46 & & & \\
\hline
\end{tabular}

interest since it would seem intuitive that patterns presented horizontally would be easier than patterns presented vertically because the children were all able to read in English and English is read from left to right, rather than top to bottom. This difference in orientation might at least be expected to play a role in recognizing letter patterns, producing an interaction. But, there was no interaction as well as no main effect. Perhaps this is an area that merits further investigation. Significant differences may not have emerged because there were only six letter patterns presented vertically and six presented horizontally. Those problems were not purely about vertical or horizontal presentation but included other parameters such as different position of the missing item as well as different numbers of items skipped in the pattern. If there is in fact a significant difference it may have been obscured. It also seems intuitive that patterns comprised of numbers would be easier in the vertical presentation since mathematics problems are usually taught presented vertically as in the case of a typical addition or subtraction problem. Again, the lack of a significant difference may be due to having only six number patterns in each orientation and the number of other factors involved. It may also be true that, because opposite effects of orientation might be expected for letter and number patterns, any differences may have offset each other. Maybe future research should compare letters and numbers presentated both vertically and horizontally without any extraneous factors.

The differences between patterns that skipped one item and patterns that skipped two items were significant. This finding may support the idea that patterning develops after the ability for seriation but preceeds or occurs concurrently with the 
mastery of transitivity. A child who is capable of solving patterns that skip one item would be able to think beyond the comparison needed in a seriation problem and may be able to make the leap of comparison needed in a simple transitivity problem. Problems that skip two items require an individual to understand the relationship between the items presented and mentally be able to fill in not just what would come immediately after the last item but what would come even further along in the sequence.

The position of the missing item (beginning, middle or end) was not shown to be significant. However when the pattern involved skipping two items rather than just one, the difference between the positions of the missing item approached significance $(.10 / p / .05)$. In the cases of problems skipping two items scores were higher in absolute values for the middle position than for the other two positions. It would be premature to accept the null hypothesis in this case. The fact that these findings approached significance makes them worth a second look. Perhaps problems in which dimension as well as orientation were not factors, allowing the children to focus solely on the number of items skipped and the position of the missing item would yield a significant difference.

In summation, the dimension in which a pattern is presented is of no consequence, but the number of items skipped in a pattern does make a difference. Patterning instruction should therefore use any dimension and first teach children patterns that skip a single item and then patterns that skip two or more items. Further research should be conducted with patterns that differ in only one or two characteristics rather than designs which involve all factors. One possibility would be to vary only the number of items skipped and the position of the missing item, to determine how the number of items skipped in a pattern interacts with the position of the missing item. Differences due to orientation may emerge if patterns involving only letters or only numbers are compared. Finally, differences due to such factors might be compared with patterns that followed different rules than those employed in this research.

\section{Author Note}

This research was conducted in partial completion of requirements for the doctoral degree by K. Marinka Gadzichowski. It was supported by grant R305A90353 from the Cognition and Student Learning Branch of the Institute of Education Sciences, US Department of Education. The opinions expressed are those of the author and do not represent views of the Institute of the US Department of Education.

The author express her appreciation for the assistance of Dr. Robert Pasnak and the gracious cooperation of Dr. Monte Dawson and the participation of the Alexandria City Public
Schools.

\section{REFERENCES}

Boyer, C., Sweeting, A., Pasnak, R., \& Kidd, J. (2010). How predictable are sequences of time, rotation, letters and numbers? Annual Convention of the Association for Psychological Science, Boston.

Burton, G. M. (1982). Patterning: Powerful play. School Science and Mathematics, 82, 39-44. doi:10.1111/j.1949-8594.1982.tb17161.x

Clements, D. H., \& Sarama, J. (2007). Effects of a preschool mathematics curriculum: Summative research on the Building Blocks Project. Journal of Research in Mathematics Education, 38, 136-163.

Clements, D. H., \& Sarama, J. (2008). Curriculum focal points for prekindergarten through grade 8 mathematics. Teaching Children Mathematics, 14, 361-365.

Cohen, J. (1992). A power primer. Psychological Bulletin, 112, 155159. doi:10.1037/0033-2909.112.1.155

Ducolon, C. K. (2000). Quality literature as a springboard to problem solving. Teaching Children Mathematics, 6, 442-446.

Economopolous, K. (1998). What comes next? The mathematics of patterning in kindergarten. Teaching Children Mathematics, 5, 230233.

Gadzichowski, K. M., \& Pasnak, R. (2010). How odd is that? Meeting of Association for Psychological Science, Boston.

Gadzichowski, M. K., Kidd, J. K. Pasnak, R., \& Boyer, C. (2010). Children's understanding of series rules. Annual Convention of the Association for Psychological Science, Boston.

Hendricks, C., Trueblood, L., \& Pasnak, R. (2006). Effects of teaching patterning to first graders. Journal of Research in Childhood Education, 21, 77-87.

Hendricks, C., Trueblood, L., Willson-Quayle, A., Malabonga, V., Ciancio, D., \& Pasnak, R., (1999). Effects of instruction in sequencing and class inclusion for first graders. Genetic, Social, and General Psychology Monographs, 125, 297-312.

Herman, M. L. (1973) Patterning before mathematics in kindergarten. Doctoral dissertation, New York: Columbia University. Dissertation Abstracts International, 33, 4060. doi:10.1080/02568540609594580

Jarboe, T. \& Sadler, S. (2003) It's as easy as 123: Patterns and activities for a creative, balanced math program. Peterborough, NJ: Crystal Springs Books.

Liljedah, P. (2004). Repeating pattern or number pattern: The distinction is blurred. Focus on Learning Problems in Mathematics, 26, $24-$ 42.

National Councils of Teachers of Mathematics (1993). Curriculum and evaluation standards for school mathematics Addenda Series, Grades K-6. Reston, VA: NCTM.

Papic, M. (2007). Promoting repeating patterns with young childrenMore than just alternating colors. Australian Primary Mathematics Classroom, 12, 8-13.

Piaget, J. (1963) The origins of intelligence in children. New York: W.W. Norton \& Company, Inc.

Threlfall, J. (1999). Repeating patterns in the early primary years. In A. Orton (Ed.), Patterns in the teaching and learning of mathematics (pp. 18-30). London: Cassell. 